\title{
A novel dermoscopic feature in traumatic onycholysis
}

\section{Ahu Yorulmaz, Basak Yalcin}

\author{
Department of Dermatology, Ankara Numune Research and Education Hospital, Ankara, Turkey
}

Corresponding author: Dr. Ahu Yorulmaz, E-mail: ahuyor@gmail.com

\begin{abstract}
Dermoscopy has certainly had a profound impact on the clinical diagnosis and management of both pigmented and nonpigmented skin lesions. Over the years, there has been much emphasis on the use of dermoscopy for the diagnosis of onychopathies. Traumatic onycholysis has been considered as one of the most common onychopathies. Although history and clinical examination remain the most crucial steps, dermoscopy apppears to be a rapid and effective tool in establishing the diagnosis of traumatic onycholysis. Here, we present two cases of traumatic onycholysis with classical and novel dermoscopic findings.
\end{abstract}

Keywords: Trauma; Onycholysis; Dermoscopy

\section{INTRODUCTION}

The nail plays an essential role in appearance. Moreover, the nail provides a barrier and protects the distal phalanx $[1,2]$. Traumatic nail diseases are a common group of nail disorders. Among them traumatic onycholysis is one of the mostly encountered one, which has been defined as the traumatic separation of the nail plate from the nail bed due to disruption of the onychodermal band $[1,3,4]$. Although history and clinical examination remain fundamental steps, dermoscopy has offered a new approach in the diagnosis of traumatic onycholysis [5-7]. Here, we present two cases of traumatic onycholysis with classical and novel dermoscopic features.

\section{Case 1}

A 19-year-old woman came to our outpatient clinic with a six months' history of thickened toenails. She had a history of working as a saleswoman and wearing pointed toed high heeled shoes for long periods of time. Dermatological examination of the patient revealed subungual hemorrhage, subungual hyperkeratosis and onycholysis in the right second toenail and bilateral great toe nails. Beau's lines also observed in the left great toe nail. Nail plate dermoscopy showed homogenenous pigmentation with peripheral fading and satellite globules, linear edged onycolytic areas and horizontal grooves (Figs. 1 and 2). Moreover, distal free edge dermoscopy of the left great toe nail revealed red and black filamentous structures in the subungual area (Fig. 3).

\section{Case 2}

A 59-year-old woman came to our outpatient clinic with a two months' history of separation of the right great toenail plate from the nail bed. She had a history of regular long distance walking for physical activity. Dermatological examination revealed distal onycholysis in the right great toenail. Nail plate dermoscopy demonstrated distal onycholysis with a linear proximal border. In addition, distal free edge dermoscopy of the right great toenail revealed reddish-black globules, black dots, red and black filamentous structures (Figs. 4- 6). Based on history, clinical and dermoscopic findings we made the diagnosis of traumatic onycholysis in both of our patients. Prior to the study, patient gave written consent to the examination and biopsy after having been informed about the procedure.

\section{DISCUSSION}

Onycholysis is a common problem encountered by dermatologist. Greatest majority of cases are caused by

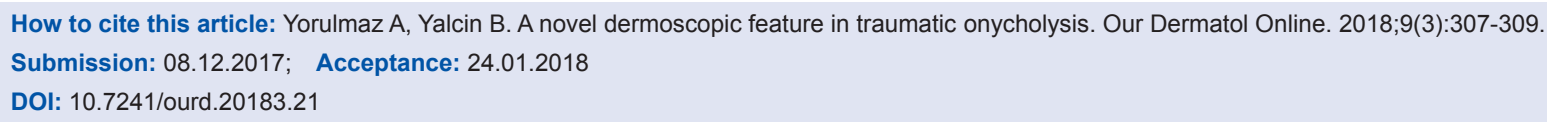




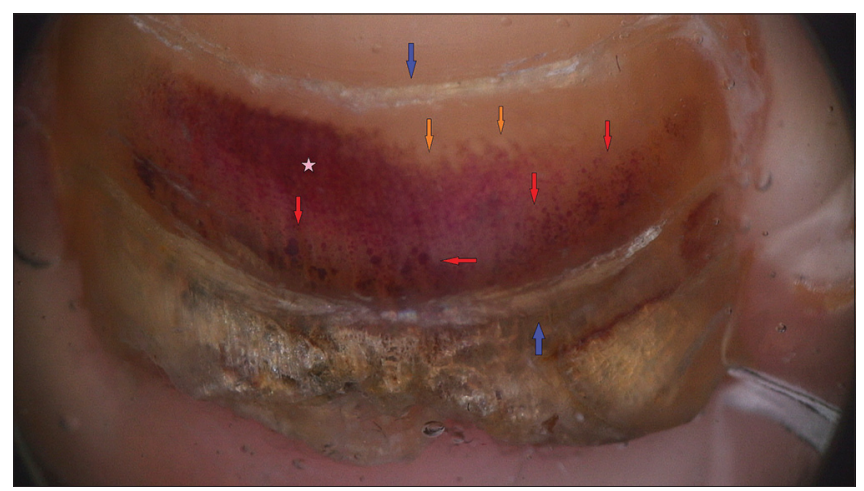

Figure 1: Nail plate dermoscopy showing reddish-purple homogeneous pigmentation (pink star) with peripheral fading (orange arrows) and satellite globules (red arrows), note linear edged distal onycholysis and horizontal grooves, which correspond to Beau's lines (blue arrows) (x20).

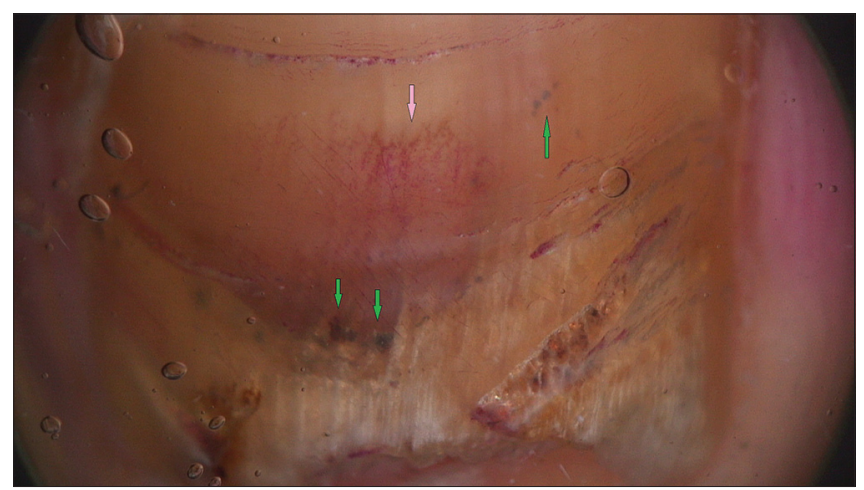

Figure 2: Dermoscopy demonstrating destruction of the nail plate and distal onycholysis. Note black globules (green arrows) and brownish red dots (pink arrow) corresponding to subungual hemorrhage (x20).

physical trauma. Generally patients are asymptomatic until they notice the whitish appearance of the transparent onycholytic nail plate [4]. "Roller coaster nails" has been defined as the typical presentation of onycholysis. This appearance describes linear edged onycholytic nail plate with an oscillating pattern [1]. Dermoscopy has been shown to be a reliable tool in differentiating traumatic onycholysis from other causes of onycholysis, which mainly include psoriasis and onychomycosis. In traumatic onycholysis the line of detachment of the plate from the bed is linear, regular and smooth and is surrounded by a normally pale pink bed. Whereas, in onychomycosis the line of detachment has a jagged appearance with sharp indentations, which are called as spikes. In psoriasis, the edge of the detachment is surrounded by an erythematous border [6,7].

In traumatic lesions not only onycholysis, but also other signs of trauma are detected. Generally, dermoscopy also reveals hemorrhagic spots and streaks, which are the main clues of subungual hemorrhage [6-8]. Onycholysis and subungual hemorrhage were the main findings in

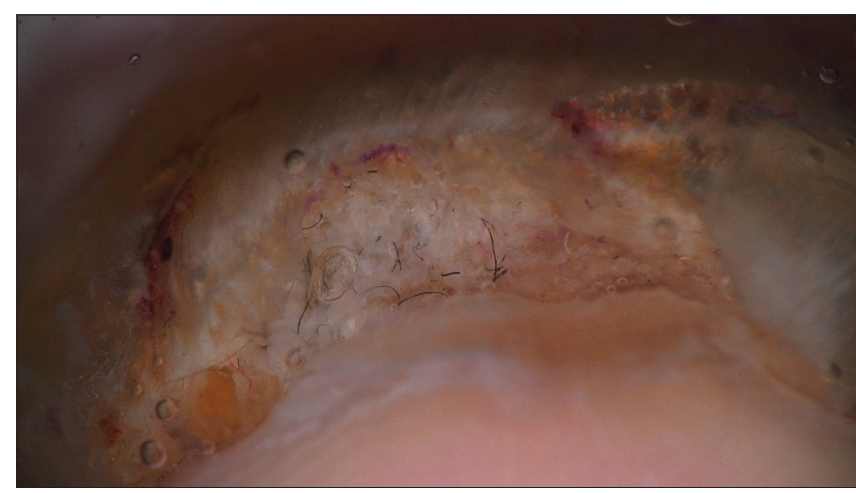

Figure 3: Dermosocpy of the distal free edge revealing subungua hyperkeratosis and red and black filamentaous structures in the hyperkeratotic area $(x 30)$.

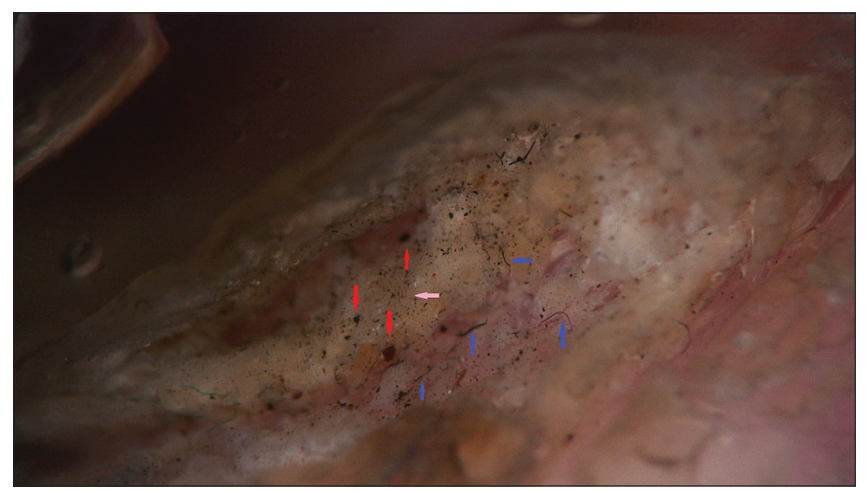

Figure 4: Distal free edge dermoscopy of the right great toenail showing subungual hyperkeratosis, reddish-black globules (red arrows), black dots (pink arrow), red and black filamentous structures (blue arrows) (x40).

our patients. Therefore, the major dermoscopic features were linear edged onycolytic areas and homogenenous pigmentation with peripheral fading and satellite globules. On the other hand, we noticed a peculiar feature in both of our patients. Distal free edge dermoscopy of traumatic nails of the patients revealed red and black filamentous structures. In one of our patients we also observed reddish-black globules and black dots.

The nail unit has a complex and abundant vascular network. It has been shown that the superficial capillary network of this complex microvasculature varies according to specialized areas of the nail unit. The hyponychium, the most distal region of the nail bed, is a specialized area, which seals the subungual space and allows the nail plate to physiologically detach from the nail bed. The subungual region of the nail unit is supplied by distal and proximal subungual arcades. The nail bed has numerous capillary loops arising from a deeper regular arrangement of sagittally aligned, parallel rows of vessels. The size of the capillary loops becomes longer and gets more inclined distally, with the longest capillary loops seen at the hyponychium $[2,9,10]$. 


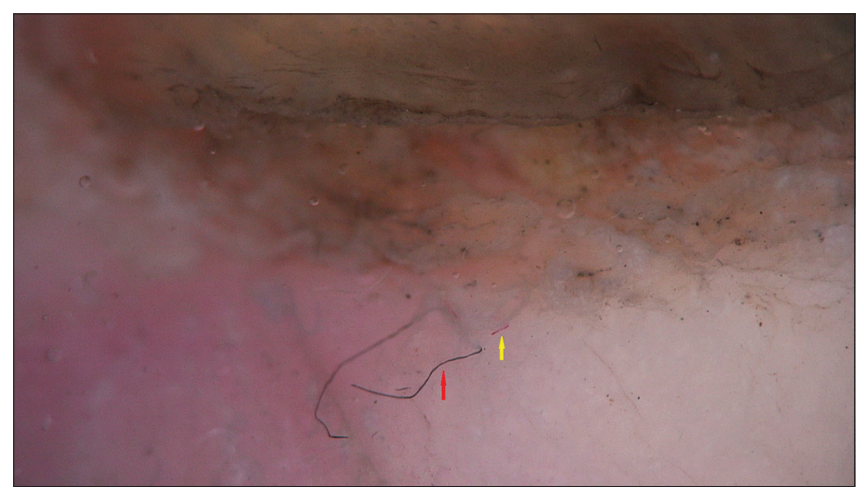

Figure 5: Dermoscopy of the hyponychium demonstrating juxtaposing black (red arrow) and red (yellow arrow) filamentous structures, note that the central region of the black filamentous structure is underneath the surface $(x 40)$.

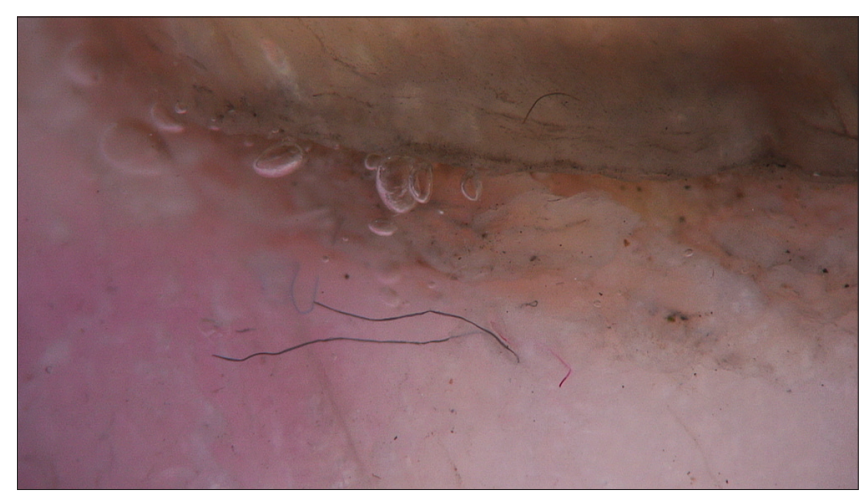

Figure 6: Altered orientation of the black filamentaous structure with changing the camera probe position, note juxtaposing red filamentaous structure $(\mathrm{x} 60)$.

In our opinion, red and black filamentous structures, that we detected on dermoscopy, correspond to subungual capillary remnants of this complex microvasculature. In a recent study, it has been demonstrated that identical dermoscopic features are found in nail bed psoriasis [11]. Nail psoriasis is characterized by increased hyponychial capillary density. Dilated, tortuous hyponychial capillaries are found in psoriatic patients $[11,12]$. Traumatic onycholysis is another nail disorder, in which damaged capillaries are exposed in the hyponychial area. Moreover, as it is seen in Figure 4, we have detected dots and globules. We suggest that these dots and globules represent the tops of the capillary loops, which are perpendicular to the surface, reclining vertical to the plane of the nail bed.

Here, we present two patients with traumatic onycholysis. Other than classical features, we have described a novel dermoscopic finding. Dermoscopy has opened new perspectives in the diagnosis of countless number of dermatological diseases. There is a growing body of literature on the use of dermoscopy as a diagnostic tool in everyday practice. On the other hand, further studies are needed to better characterize unusual dermoscopic findings.

\section{CONSENT}

The examination of the patient was conducted according to the Declaration of Helsinki principles.

\section{REFERENCES}

1. Rieder EA, Tosti A. Cosmetically induced disorders of the nail with update on contemporary nail manicures. J Clin Aesthet Dermatol. 2016;9:39-44.

2. Haneke E. [Anatomy, biology, physiology and basic pathology of the nail organ].Hautarzt. 2014;65:282-90.

3. Bharathi RR, Bajantri B. Nail bed injuries and deformities of nail. Indian J Plast Surg. 2011;44:197-202.

4. Zaias N, Escovar SX, Zaiac MN. Finger and toenail onycholysis. J Eur Acad Dermatol Venereol. 2015;29:848-53.

5. Elfar NN, Abdel-Latif AM, Labeh EA. Role of onychoscopy in differentiation between distal subungual onychomycosis, psoriasis, and traumatic onycholysis. J Egypt Women Dermatol Soc. 2015;12:145-9.

6. Alessandrini A, Starace M, Piraccini BM. Dermoscopy in the Evaluation of Nail Disorders. Skin Appendage Disord. 2017;3:70-82.

7. Piraccini BM, Bruni F, Starace M. Dermoscopy of non-skin cancer nail disorders. Dermatol Ther. 2012;25:594-602.

8. Mun JH, Kim GW, Jwa SW, Song M, Kim HS, Ko HC, et al. Dermoscopy of subungual haemorrhage: itsusefulnessindifferentialdiagnosis from nail unit melanoma. Br J Dermatol. 2013;168:1224-9.

9. Hasegawa K, Pereira BP, Pho RW. The microvasculature of the nail bed, nail matrix, and nail fold of a normal human fingertip. J Hand Surg Am. 2001;26:283-90.

10. de Berker D. Nail anatomy. Clin Dermatol 2013;31:509-15.

11. Yorulmaz A, Artuz F. A study of dermoscopic features of nail psoriasis. Postepy Dermatol Alergol. 2017;34:28-35.

12. Iorizzo M, Dahdah M, Vincenzi C, Tosti A. Videodermoscopy of the hyponychium in nail bed psoriasis. J Am Acad Dermatol. 2008;58:714-5.

Copyright by Ahu Yorulmaz, et al. This is an open access article distributed under the terms of the Creative Commons Attribution License, which permits unrestricted use, distribution, and reproduction in any medium, provided the original author and source are credited.

Source of Support: Nil, Conflict of Interest: None declared. 